\title{
Modeling of molecular reorientation in nematic liquid crystals
}

\author{
Filip A. Sala, ${ }^{* 1}$ Maksymilian J. Bujok, ${ }^{2}$ Mirosław A. Karpierz ${ }^{1}$ \\ ${ }^{1}$ Faculty of Physics, Warsaw University of Technology, Koszykowa 75, 00-662 Warszawa, Poland \\ ${ }^{2}$ Faculty of Mathematics and Information Science, Warsaw University of Technology, Koszykowa 75, \\ 00-662 Warszawa, Poland
}

Received March 02, 2016; accepted March 17, 2016; published March 31, 2016

\begin{abstract}
In this paper, the modeling of molecular reorientation in nematic liquid crystals is described. The theoretical model is based on Frank-Oseen elastic theory. By minimizing the equation on free energy, an equation describing molecular reorientation is obtained. To get a solution, two numerical methods (Successive Over-Relaxation and Multigrid) are employed and compared for the numerical results of director orientation and computation time.
\end{abstract}

Nematic liquid crystals are unique materials commonly used, for instance, in many types of displays. These devices are based on strong nonlinear properties of liquid crystals. The most important mechanism is reorientational nonlinearity as the molecules react to the electric and magnetic fields and change their average orientation i.e. director $\boldsymbol{n}$. To describe the optical properties of liquid crystals it is crucial to model molecular reorientation as it influences refractive indexes and absorption which strongly affect light propagation in such materials [1-3]. However, there are some analytical solutions of molecular reorientation for simplified cases [4-5]; in more complex configurations, numerical methods have to be employed. In this article, comparison of two numerical methods, used to solve the same equation based on elastic theory, is presented.

Let us consider a cell of length $\mathrm{L}$, filled with a liquid crystal. The director is defined as:

$$
\vec{n}=[\cos \varphi, \sin \varphi] .
$$

The molecules lie in the $x y$ plane and the orientation is defined by an angle $\varphi$ (see Fig. 1). On both sides of the cell, fixed anchoring conditions are applied:

$$
\varphi(0)=\varphi_{0}, \quad \varphi(L)=\varphi_{1} .
$$

Substituting director $\boldsymbol{n}$ into Frank-Oseen equation on free energy:

\footnotetext{
*E-mail: sala@if.pw.edu.pl
}

$$
\begin{aligned}
& f=\frac{1}{2} \mathrm{~K}_{11}(\nabla \vec{n})^{2}+\frac{1}{2} \mathrm{~K}_{22}(\vec{n} \cdot(\nabla \times \vec{n}))^{2}+ \\
& +\frac{1}{2} \mathrm{~K}_{33}(\vec{n} \times(\nabla \times \vec{n}))^{2}-\frac{1}{2} \Delta \varepsilon \varepsilon_{0}(\vec{n} \cdot \vec{E})^{2},
\end{aligned}
$$

and minimizing it, by using Euler-Lagrange equations and assuming single constant approximation, for onedimensional case leads to the following equation [6-7]:

$$
\frac{\partial^{2} \varphi}{\partial x^{2}}+\frac{\Delta \varepsilon \varepsilon_{0}}{2 \mathrm{~K}}\left|E_{y}\right|^{2} \sin 2 \varphi=0
$$

where: $E_{y}$ - electric field distribution, K - average Frank elastic constant of the material, $\Delta \varepsilon$ - electric anisotropy, $\varepsilon_{0}$ - electric constant.

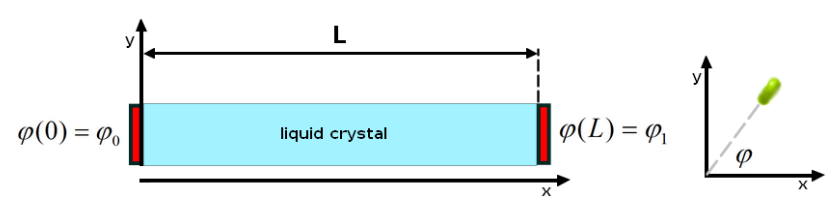

Fig. 1. The analyzed setup (left) and coordinate system (right).

To solve Eq.(2), two numerical methods were used. The first is the Successive Over-Relaxation method [8] and the second - multigrid method [9]. Both methods are compared for molecular reorientation and computation time.

The Successive Over-Relaxation algorithm is based on the Gauss-Seidel scheme with the relaxation parameter $\omega=1.7$ and constant resolution $\mathrm{h}=0.1 \mu \mathrm{m}$. In the presented results, 30000 of iterations were made. The SOR scheme can be written as:

$$
\begin{aligned}
& \varphi^{(n+1)}(x)=\left[\frac{\varphi^{(n)}(x+h)+\varphi^{(n+1)}(x-h)}{2}+\right. \\
& \left.+\frac{\beta h}{2} \sin 2 \varphi^{(n)}(x)\right] \cdot \omega+(1-\omega) \cdot \varphi^{(n)}(x),
\end{aligned}
$$


where $\beta=\frac{\Delta \varepsilon \varepsilon_{0}}{2 \mathrm{~K}}\left|E_{y}\right|^{2}, n-$ denotes iteration.

The multigrid method is implemented with the Full Approximation Scheme (FAS) in a two-grid form [10-11] which is a generalization of multigrid algorithms. The algorithm is based on calculations on a coarser grid (which is faster) and then corrections are interpolated and applied to the results. The two-grid method can be also described as a combination of a coarse grid method and that of relaxation.

Fig. 2 presents the numerical results of molecular reorientation under the influence of a constant electric field coming from the optical beam. The parameters used were as follows: $\Delta \varepsilon=0.49, \mathrm{~K}=5.5 \mathrm{pN}$, which corresponds to the parameters of 6CHBT liquid crystal illuminated with a wide optical beam of a wavelength of $793 \mathrm{~nm}$ at a temperature of $25^{\circ} \mathrm{C}$. At the boundaries, Dirichlet anchoring conditions are assumed:

$$
\varphi(0)=\varphi(L)=45^{\circ} .
$$

As the analyzed issue is a boundary value problem, the solution depends on the boundary conditions. On the other hand, the maximum reorientation depends on electric field power as well as anisotropy and elastic constant. A cell of $50 \mu \mathrm{m}$ in length is analyzed.

(a)

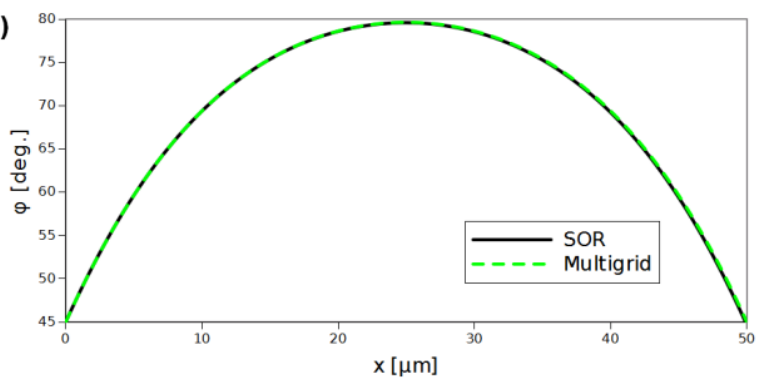

(b)

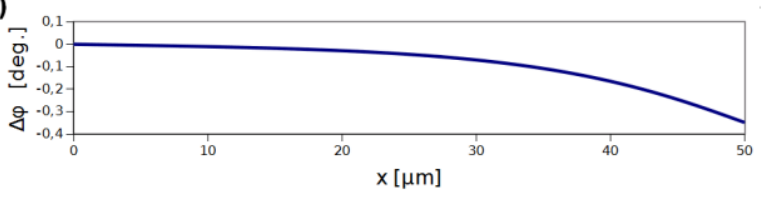

Fig. 2. (a) Comparison of molecular orientation calculated with SOR and multigrid methods. (b) Difference in the results between molecular reorientation obtained with SOR and multigrid methods.

According to Fig. 2b, it appears that differences between these two methods increase with position $x$. It is caused by the fact that both methods used to solve Eq. (3) are iterative. Even though the finite difference used to denote second derivative is symmetric, the calculations are made from $x=0 \mu \mathrm{m}$ up to $x=\mathrm{L}=50 \mu \mathrm{m}$ so the error cumulates. In spite of that the molecular reorientation should be symmetric but in fact it is not. There are some slight differences which are of an order lower for the multigrid algorithm than for the SOR method.

To compare the correctness of the results, the energy of both solutions was calculated. The lower the energy the more correct are the results. In a discrete form, the normalized energy in one dimension can be expressed as:

$$
Q=\sum_{i}\left(\left[\frac{\varphi(i+1)-\varphi(i-1)}{2 h}\right]^{2}-\frac{\Delta \varepsilon \varepsilon_{0}}{\mathrm{~K}} E_{y}{ }^{2}(i) \sin ^{2} \varphi(i)\right),
$$

where $i$ - index corresponding to the discretized $x$ position.

For the presented results, the energy of the system is as follows: $\mathrm{Q}=-2.9209 \mathrm{arb}$.u. for the SOR method, and $\mathrm{Q}=$ -2.9285 arb.u. for the multigrid method. It shows that using the multigrid method it is possible to obtain slightly better results.

The computation time of both methods was also analyzed. The time needed to obtain the solution of assumed energy is presented in Fig. 3 for both methods. For very low system sizes, the computation time is similar but for the SOR method, it quickly rises with the grid size. It shows the advantage of the multigrid method for large systems.

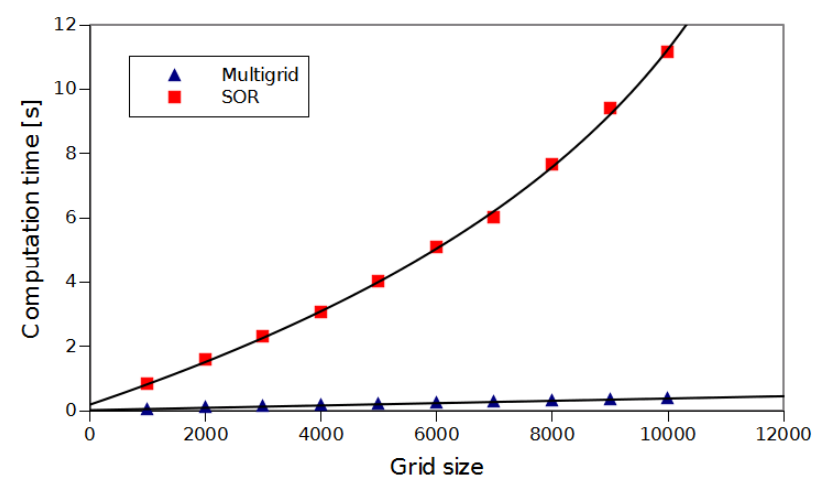

Fig. 3. Comparison of computation time for both numerical methods as a function of grid size.

Concluding, in the following article two iterative methods were used to solve the equations describing molecular reorientation of nematic liquid crystal. Both methods, Successive Over-Relaxation and multigrid, based on the Full Approximation Scheme in a two-grid form gave very similar results. The multigrid method proved to give slightly more symmetric results and better solutions when comparing the energy of the obtained results. Moreover, the multigrid method has lower computation times, especially for large systems. On the other hand, the SOR method, which produced good results, is straightforward and easier to use than the multigrid method, especially for more complicated nonlinear equations. To obtain good results in an easy 
way, the SOR method can be used. However, when very accurate results are needed and the computation time is crucial, some effort has to be made to implement the multigrid method.

This work was supported by the grant of the Faculty of Physics at the Warsaw University of Technology.

\section{References}

[1] G. Assanto, M.A. Karpierz, Liq. Cryst. 36, 1161 (2009).

[2] G.D. Ziogos, E.E. Kriezis, Opt. Quant. Electron. 40, 10 (2008)

[3] M. Kwaśny, U.A. Laudyn, F.A. Sala, A. Alberucci, M.A. Karpierz, G. Assanto, Phys. Rev. A 86, 013824 (2012).

[4] M.A. Karpierz, Phys. Rev. E 66, 036603 (2002).

[5] A. Walczak, Proc. SPIE 4759, 327 (2002).

[6] F.A. Sala, M.A. Karpierz, J. Opt. Soc. Am. B 29, 1465 (2012).

[7] F.A. Sala, M.A. Karpierz, Opt. Express 20, 13923 (2012).

[8] D.M. Young, Iterativemethods for solving partial difference equations of elliptical type, Ph.D. thesis (Harvard University, 1950).

[9] D. Kincaid, W. Cheney Numerical Analysis: Mathematics of Scientific Computing (American Mathematical Society, 2002).

[10] W.L. Briggs , V.E. Henson, S.F. McCormick A Multigrid Tutorial, (Society for Industrial and Applied Mathematics, 2000).

[11] A. Brandt, O. Livne, Multigrid Techniques (Society for Industrial and Applied Mathematics, 2011). 